Bladder cancer is one of the most common malignancies worldwide. The transurethral resection of bladder tumour (TURB) remains the gold standard in both diagnostics and treatment. Because of the importance of TURB in bladder cancer management and the fact that TURB is one of the most commonly performed urologic procedures, it is the subject of continuous technological development. The latest advances in the field of endourology are aimed at increasing surgical accuracy and thus reducing the risk of bladder tumour recurrence and progression. However, despite the constant progress in technology and technique, there are still a lack of good quality data showing the superiority of any of the methods. The aim of this paper is to present available data on new technological developments in surgical technique of TURB. Advantages and disadvantages of currently available methods are discussed, and literature showing their effectiveness and safety is shown.

Key words: bladder cancer, transurethral resection, en bloc.

Contemp Oncol (Pozn) 2019; 23 (4): 195-201 DOI: https://doi.org/10.5114/wo.2019.91530

\section{Technical developments in transurethral resection of bladder tumours}

\author{
Agata Pastuszak ${ }^{1}$, Romuald Zdrojowy ${ }^{1}$, Stawomir Poletajew ${ }^{2}$, \\ Jan Adamowicz ${ }^{3}$, Wojciech Krajewski ${ }^{1}$
}

\author{
${ }^{1}$ Department of Urology and Oncological Urology, Wroclaw Medical University, \\ Wroclaw, Poland \\ 2Department of General, Oncological, and Functional Oncology, Medical University \\ of Warsaw, Warsaw, Poland \\ ${ }^{3}$ Department of Regenerative Medicine, Ludwik Rydygier Collegium Medicum \\ in Bydgoszcz, Nicolaus Copernicus University, Torun, Poland
}

\section{Introduction}

Bladder cancer (BC) is the ninth most common malignancy worldwide with a yearly incidence of approximately 430,000 cases, and it is positioned $13^{\text {th }}$ in terms of yearly oncological mortality [1]. It is estimated that 3/4 of primary diagnosed bladder tumours present as non-muscle invasive cancers (NMIBC) - a group comprising lesions limited to bladder mucosa (stage Ta and CIS) and infiltrating submucosal layer of the bladder wall (stage T1). Due to non-advanced stage, correctly managed NMIBCs present relatively good survival rates, but with a high perpetual risk of tumour recurrence or progression to muscle invasive disease. Therefore, precise and meticulous diagnostic and therapeutic proceedings must be employed.

It must be emphasised that both NMIBC diagnosis and treatment rely on endoscopic transurethral resection of bladder tumour (TURB). Because of the importance of TURB in BC management and the fact that TURB is one of the most commonly performed urologic procedures, it is the subject of continuous technological development.

The aim of this paper is to present available data on new technological developments in the surgical technique of TURB.

\section{Evidence acquisition and evidence synthesis}

A literature search according to PRISMA guidelines within the Medline database was conducted in December 2018 for papers presenting the technical developments of TURB, without setting time limits, using the terms "bladder cancer" in conjunction with TURB, monopolar, bipolar, plasmakinetic, laser, and en bloc. Boolean operators (NOT, AND, OR) were also used in succession to narrow and broaden the search (Fig. 1). AutoAlerts in Medline were also run, as well as reference lists of original articles and review articles for further eligible data. Additionally, resource centres from the largest urological conferences were searched. The search was limited to English, Polish, German, and Spanish literature. Articles that did not address the topics were excluded, and the full text of the remaining articles was subsequently reviewed.

\section{Discussion}

\section{Conventional resection}

The procedure of transurethral tumour resection was first performed and described by Edwin Beer in 1910 [2]. From that moment, despite the technological advancement in the subsequent 100 years, the main idea of TURB remained the same. According to European Association of Urology (EAU) 


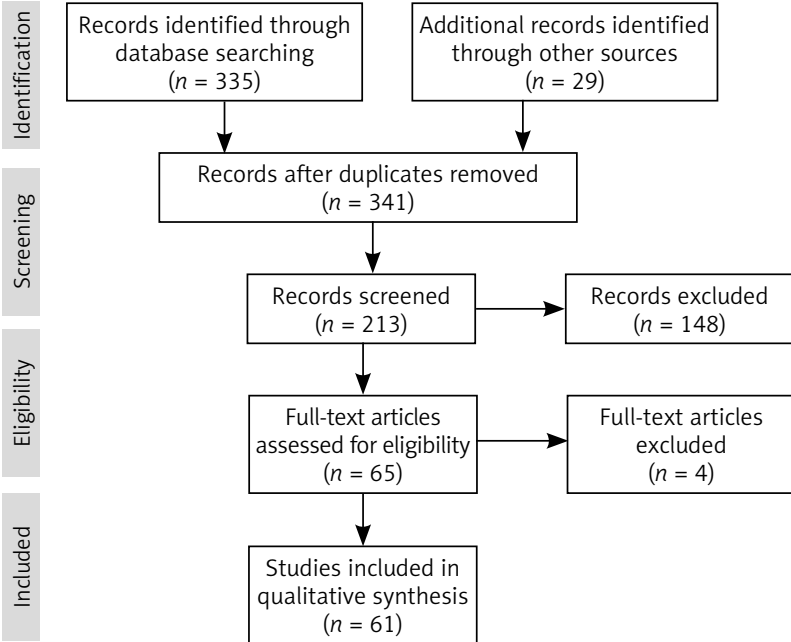

Fig. 1. The flow of information through the different phases of the systematic review (adopted from www.prisma-statement.org)

guidelines, the goal of TURB in Ta and T1 BC is to make the correct diagnosis and completely remove all tumours with appropriate muscular layer sampling. In muscle-invasive disease radical excision is often impossible, and therefore the most important objective is an accurate diagnosis based on the analysis of muscle invasion.

The gold standard for transurethral resection of bladder tumours is conventional TURB (CTURB), classically performed with monopolar current (mTURB). The CTURB is widely used, and therefore tremendous experience in this procedure been accumulated over the years. The procedure is performed in the lithotomy position, mainly under spinal anaesthesia. However, because of lack of muscular relaxation, and the fact that during monopolar electrocautery the electric current runs from the resection loop through the patient's body to the grounding pad placed on the patient's skin, there is a high risk of obturator nerve stimulation (obturator reflex). Due to abrupt adductor muscle contraction, accidental perforation of the bladder wall with thermal damage of adjacent tissue may occur. Additionally, when monopolar energy is applied, the electrical resistance generates a high temperature (up to 300$400^{\circ} \mathrm{C}$ ) with significant collateral and penetrative tissue injury, which might hamper deeper resection and result in a significant charring of the specimen tissues leading to cancer mis-staging or mis-grading [3]. Finally, mTURB is performed in a nonconductive irrigative solution (water, glycine, sorbitol, or mannitol). The excessive absorption of this fluid into the opened vessels and subsequent fluid and electrolyte abnormalities may cause the life-threatening TUR syndrome.

To overcome those shortcomings, bipolar current was introduced in BC resections (bTURB). In this setting, isotonic saline is used as the irrigant, and the electric current runs between two electrodes incorporated in the resectoscope. Highly ionised particles (plasma field) created around the resection loop disrupt the organic molecular bonds between tissues, generating much lower temperatures $\left(40-70^{\circ} \mathrm{C}\right)$ when compared to monopolar energy [4, 5]. Because of those facts, the resection with bipolar ener- gy is more precise with better haemostatic and less charring effect on both the resection bed and histopathological samples. Additionally, bTURB is a safer option for patients with implanted pacemakers or pregnant women [6].

To date, various authors have tried to compare monopolar resection with newer, bipolar resection, but with conflicting results. In the paper by Del Rosso et al. $132 \mathrm{pa}$ tients were randomised to undergo mono- or bipolar resection of primary NMIBC. The authors demonstrated that bTURB represents a safe and effective procedure in the management of NMIBC; however, the study does not present any advantage between study groups apart from hospitalisation and catheterisation times [7]. Another randomised study included 147 patients and showed statistical differences in the incidence of obturator reflex and lower incidence of severe cautery artefacts in the bipolar arm. Nevertheless, there were no significant differences in other analysed variables [8]. In the study analysing quality of bladder detrusor muscle sampling by Teoh et al., the authors randomised 160 patients to both procedures and noted that bTURB was significantly associated with better muscle sampling, with an odds ratio (OR) of 2.23 . Additionally, fewer patients in the bTURB group required postoperative irrigation when compared to patients in the monopolar arm [9]. In another prospective, randomised analysis, 130 patients underwent mono- or bipolar TURB. The authors did not find any statistical differences in tumour resection completeness, muscle sampling, or thermal specimen damage rates. However, obturator reflex was experienced in $21.5 \%$ of the patients in the monopolar TURB group and in $4.6 \%$ of the patients in bTURB group. Additionally, the number of bladder perforations was significantly higher in mTURB (21.5\% vs. 6.1\%) [10]. These observations were confirmed in a large retrospective analysis of perioperative outcomes of 8188 mono-bipolar pairs. Sugihara et al. showed that bTURB was associated with a substantially lower incidence of perioperative complications, including severe bladder injury [11]. On the other hand, another retrospective TURB complication analysis on 586 procedures by Avallone et al. did not show any statistically significant differences between investigated procedures. The authors emphasised that obturator reflex and significant adductor contraction can also occur during bTURB. Similarly, a post-hoc analysis from a randomised trial of various visualisation methods during TURB did not find any statistically significant differences between the two methods. The authors stated that bTURB seems to have no evident advantages over mTURB with respect to operation time, perioperative and postoperative complication rates, and recurrence rates at 12 months [12].

Finally, in a meta-analysis including almost 1000 patients from eight trials, bTURB was associated with shorter operative time, shorter hospital stay, less blood loss, and shorter catheterisation time. What is more, there were fewer complications such as obturator reflex and bladder perforation in the bTURB group. Also, the recurrence rates up to two years after the procedure were slightly more favourable for bTURB [6]. Nevertheless, another meta-analysis did not corroborate those findings; Cui et al. found that there were no statistically significant differences between 
bTURB and mTURB in terms of obturator reflex, bladder perforation, and transfusion rates. Also, the grade of tissue sample cautery artefacts and recurrence rate did not differ significantly [13].

In conclusion, despite extensive experience and an abundance of reports, available studies do not provided solid evidence that bipolar resection is superior to monopolar resection in terms of surgical outcome, complication risk, or tumour recurrence rate.

\section{En bloc transurethral resection of bladder tumour}

The main disadvantage of cutting wire-loop resection is the necessity to use the "incise and scatter", piece-bypiece technique. As is widely known, scattering the neoplastic tissue runs against the basic principles of oncological surgery, leading to exfoliation of numerous cancer cells. Theoretically, it may cause out-of-field recurrences by implementation of circulating cells [14]. Because the residual tumour rates after TURB concern up to $35 \%$ of cases and recurrence of up to $75 \%$ of patients, at least some of those clinical events may be caused by implementation of released cancer cells [15]. The other major problem of CTURB is the difficulty in accurately pathologically evaluating fragmented, disoriented, and heavily damaged samples. This often leads to inaccurate invasion depth and malignancy grade determination [16]. It is widely known that the presence of lamina muscularis of the bladder in the histopathological specimen is considered a surrogate criterion of the operation quality. As shown in the literature, the absence of muscle in the specimen is associated with a significant risk of residual disease, tumour misstating, and early recurrence [17].

For those reasons, the en bloc resection concept was developed. It comprises resection of the whole tumour in one piece - the exophytic papillary tumour together with the base and bladder wall fragment. When the lesion is resected in that manner, the normal histological relations of the specimen are preserved, rates of lamina muscularis presence go beyond 95\%, and the cells' dispersal is (theoretically) diminished $[18,19]$. What is more, excellent bleeding control is possible [16].

The en bloc technique differs slightly between various studies; however, the main principles include: 1) creating a circular incision up to the depth of the muscle layer at some distance from the tumour; 2) progressive dissection of the lesion within the muscular layer using both blunt dissection and some form of energy, mainly in a "flash-firing" fashion; 3) final detachment of the tumour; and 4) tumour retrieval through the resectoscope [20]. For smaller tumours one-swing loop resection or the "grasp and bite technique" can also be used [21]. Finally, thermal/laser ablation in local anaesthesia of small lesions with strong low-grade conviction may be employed, especially in an outpatient follow-up setting [22]. Nonetheless, being therapeutic enough in selected cases, fulguration cannot be classified as en bloc resection.

The major drawback of the en bloc technique is the tumour size limit, which must finally fit and be pulled in one piece through the working channel of the endoscope. Depending on the tumour morphology, cohesion, and amount of resected bladder wall, a diameter of $3 \mathrm{~cm}$ is generally considered the upper limit of tumour size that can be extracted in one piece [18].

In the available literature various retrieval methods have been described. The easiest ones comprise simple washing with or without pressure evacuators and usage of cystoscopy graspers or a thick vaporisation loop. Some authors advocate wedging the specimen in front of a resectoscope beak and subsequently removing the whole instrument through the urethra [23]. Naselli et al. describe usage of laparoscopic forceps for the retrieval of tumours as large as $4.5 \mathrm{~cm}$ [24]. Similarly, Hurle et al. used a nephroscopy sheath and a laparoscopic grasper [25]. In a paper by Maurice et al., the authors described usage of a nylon mesh retrieval net to retrieve tumours larger than $5 \mathrm{~cm}$ [26]. Other authors reported retrieval of tumours with standard endo-bags commonly used in gastroenterology [27]. Finally, some authors propose the extraction of non-compliant lesions by cutting the specimen into a few pieces [16]. However, in cases when the tumour is retrieved in front of the whole instrument, it subjectively seems that theoretical urethral seeding has to be considered so as not to compromise oncological principles.

Some other en bloc problems may be related to lesion location, with the most difficult resection in the bladder dome, anterior wall, and bladder neck. However, it has to be remembered that the CTURB also yields some risks in those locations, and as the growing experience shows - it is not smaller when compared to en bloc.

En bloc resections can be performed by means of various energy sources including electric energy and lasers.

\section{En bloc with electric energy}

The first reports of en bloc resection were delivered in the late 1990s [28, 29]. To perform the procedure, Ukai et al. used a modified J-shaped monopolar loop. The electrode was created from a traditional right-angle loop electrode with the left half of the loop cut off, and the right half trimmed to $2 \mathrm{~mm}$ and then bent inward. Later, the knife electrode was presented by Saito et al., flat loop by Lodde et al., and Collins loop by Hurle et al. [25, 30, 31]. In the literature we can also find reports about en bloc resection with classic wire loop, as well as bipolar loop or bipolar "button" [32-35].

To date, several studies comparing electric en bloc resection and classical resection were published. Sureka et al. showed in a prospective non-randomised study on 45 patients that there was a significant reduction in the recurrence rate and time to recurrence with en bloc when compared with mTURB. However, the progression rate did not differ significantly [34]. In the next study on 90 patients by Zhang et al., the authors did not show statistically significant differences in operative time, surgical complications, and recurrence rates between the groups. However, the detrusor muscle could be identified in $100 \%$ of en bloc specimens but only in 70\% of cTURB samples [35]. In other study, 90 patients were prospectively randomised to undergo bi- 
polar button en bloc or mTURB. Reduced obturator reflex rate, mean operation time, haemoglobin level drop, catheterisation period, and hospital stay were observed in the en bloc arm. Additionally, a lower recurrence rate was found in the en bloc group with decreased rate of heterotopic recurrent lesions [33]. Finally, in a meta-analysis of 886 patients (438 en bloc, 448 cTURB) Wu et al. proved that hospitalisation and catheterisation time were shorter in the en bloc group with no significant difference in operation time. Also, there was a significant difference in recurrence and complication rates in favour of en bloc resection [36].

\section{En bloc using water dissection}

One of the difficulties in en bloc resection is finding and following the right, deep plane of dissection, without perorating the bladder. This is particularly important in tumours localised in the bladder dome or posterior wall and in patients with atrophic or severe obstructive bladder.

In such cases, the well-known gastroenterological tool, a water-jet dissector, may be helpful. The main idea of the device is to create a fluid cushion underneath the tumour by injecting saline, elevate the lesion, and therefore facilitate precise and safe resection.

In the available literature only a few case series of hydrodissection usage in NMIBC are available. In those, the authors present their experience with tumours up to $7.5 \mathrm{~cm}$ in diameter [27, 37, 38]. What is worth mentioning, good quality data regarding this method are presented at international conferences. In the 2017 Société Internationale d'Urologie (SIU) conference, Hu presented single-centre, randomised, controlled trial results from 86 patients receiving either en bloc transurethral water jet resection or CTURBT. The authors proved that hydrodissection is safe and feasible; however, according to Kaplan-Meier survival curves, there was no statistical difference in the rate of recurrence at 12 months [39]. In the 2017 EAU congress, Gakis et al. presented results of a randomised, controlled trial including 115 patients. Detrusor muscle presence, complication rate, and catheterisation and hospitalisation times were not significantly different between both groups. Despite the fact that the operation time was higher in the en bloc group, the procedure was associated with significantly higher histopathologically confirmed complete resection status (pRO) [40].

\section{En bloc using a holmium laser}

The holmium laser is one of the most versatile lasers in urology. This pulsed wave laser with a wavelength of $2100 \mathrm{~nm}$ (absorbed by water) may be used for tissue ablation, cutting, coagulation, as well as lithotripsy [41]. Because of its shallow penetration depth and lack of obturator reflex, it allows for very precise tumour resection.

One of the first reports about holmium laser usage in bladder tumour resections was published by SolerMartínez et al. In the study on 36 patients operated under local anaesthesia and sedation the authors presented "photocoagulation" of the papillary bladder tumours. They observed no serious complications, and the recurrence rate was similar when compared to cTURB [42]. In next study,
Zhu et al. presented the results of 101 holmium laser resections of bladder tumours (HoLRBT) and 111 cTURBs on 212 patients with primary NMIBC. The complication rates, and irrigation and catheterisation times were lower in the HoLRBT group; however, the cTURB group had shorter operation times. It is worth mentioning that there were no differences in the recurrence-free rates between the study groups [43]. In the study by Xishuang et al. the authors retrospectively evaluated data of 51 patients who underwent MTURB, 58 patients who underwent bTURB, and 64 patients after HoLRBT. They proved that both bTURB and HoLRBT were associated with fewer intraoperative and postoperative complications, as well as less bleeding and shorter postoperative bladder irritation, catheterisation, and hospitalisation times. Once again, there were no significant differences in recurrence rates between groups [44]. In the next study on 27 patients after MTURB and 23 patients after HoLRBT, the authors showed that there were no significant differences in operation time and recurrence rates between the two groups. The HoLRBT group had lower intraoperative and postoperative complication rates, but there were no significant differences in the transfusion rates and occurrence of urethral strictures [45].

\section{En bloc using a thulium laser}

The thulium continuous wave laser was introduced in the early 2000s [46]. With a water-absorbed wavelength of $2013 \mathrm{~nm}$ (close to the peak absorption of water) and $0.25 \mathrm{~mm}$ of penetration depth, the thulium laser allows for very precise and accurate resection [47].

The first reports about thulium laser resection of bladder tumours (ThuLRBT) were presented by Yang et al. and published in a series of articles. The authors stated that resection with thulium is a safe, efficient, and effective method [48-54]. Zong et al. presented a study on 95 patients comparing thulium, holmium, and conventional resection. The authors observed a lower haemoglobin decrease after the thulium procedure, but no differences in recurrence-free survival were observed [55]. Liu et al. published the results of a prospective, randomised trial comparing ThuLRBT and CTURB. The study comprised 120 patients (56 cTURB and 64 ThuLRBT), and no significant differences in recurrence rates were shown between the groups. On the other hand, intra- and postoperative complications were less frequently observed in the laser group [56]. In another paper, Chen et al. prospectively compared 142 patients undergoing ThuLRBT and CTURB in a randomised manner. The operation times were longer when using laser, but the complication rates were lower. No differences in recurrence-free survival were noted [57]. In the randomised prospective comparison of 143 patients after CTURB and 149 after thulium vaporesection, Zhang et al. showed that operation times were shorter and complication rates higher with CTURB, and there were no differences in recurrence-free and progression-free survival [58]. Migliari et al. compared a prospective group of $58 \mathrm{pa}$ tients who underwent ThuLRBT with a historical cohort of 61 patients after mTURB. The authors reported lower complication rates and higher rates of lamina muscularis in the 
specimens from the laser group [59]. In the retrospective study on 256 patients by Li et al., comparing ThuLRBT and bTURB, the authors showed that operation and hospitalisation times, and postoperative irrigation and catheterisation durations were significantly shorter in the thulium group. Also, the rate of bladder detrusor muscle identified in pathologic specimens was higher in patients operated by laser. However, no differences in fever, bleeding, and recurrence-free rates were observed [60].

Lately, a second generation of thulium laser has been introduced in the NMIBC setting (1.9 $\mu \mathrm{m}$ Vela laser) [61]. In the study by Xu et al. the authors compared retrospectively data of 26 patients after ThuLRBT and 44 after CTURB. They observed lower complication rates and better quality of histopathological specimens when using laser; however, there were no differences in procedure duration and transfusion and recurrence-free rates [62].

\section{En bloc using a green-light KTP laser}

The first report on green-light laser $(532 \mathrm{~nm})$ usage in the NMIBC setting was presented by Tao et al. in the late 1990s. The authors published data comparing 74 patients who underwent laser vaporisation and 84 patients after CTURB. No significant differences were observed in operative time and perioperative and postoperative serum sodium and haemoglobin levels. The green laser was associated with fewer complications, and the recurrence-free rates were lower. Yet, the main disadvantage was the lack of sufficient tissue for pathologic examination [63]. Subsequently, He et al. introduced en bloc with a KTP laser using front-firing fibre on 45 patients, showing the feasibility and safety of the procedure [64]. In the prospective, non-randomised study on 158 patients (83 KTP en-bloc and 75 CTURBT) by Chen et al. the authors showed that resection with laser was associated with lower complication rates and had shorter duration. Still, the recurrence-free survival rate did not differ significantly between the two groups after 36 months of follow-up [65].

One feature of green laser should be emphasised. The KTP laser energy is absorbed by haemoglobin, which results in excellent haemostatic abilities. Hence, the green laser procedure may be performed under local anaesthesia in patients who are under oral anticoagulation. Clearly not being an option for every-day practice, it may be helpful in some carefully selected patients.

\section{Conclusions}

In recent years, the technological development in urological surgery has been remarkable. The latest advances in the field of endourology are aimed at increasing surgical accuracy and thus reducing the risk of tumour recurrence and progression. However, despite the constant progress in technology and technique, there are still a lack of good quality data showing the superiority of any of the methods. It should be remembered that, regardless of the surgical method, adjuvant treatment should be implemented in accordance with the current EAU guidelines for NMIBC.

The authors declare no conflict of interest.

\section{References}

1. Antoni S, Ferlay J, Soerjomataram I, Znaor A, Jemal A, Bray F. Bladder Cancer Incidence and Mortality: A Global Overview and Recent Trends. Eur Urol 2017; 71: 96-108.

2. Beer E. Landmark article May 28, 1910: Removal of neoplasms of the urinary bladder. By Edwin Beer. JAMA 1983; 250: 1324-1325.

3. Singh H, Desai MR, Shrivastav P, Vani K. Bipolar versus monopolar transurethral resection of prostate: randomized controlled study. J Endourol 2005; 19: 333-338.

4. Puppo P, Bertolotto F, Introini C, Germinale F, Timossi L, Naselli A. Bipolar transurethral resection in saline (TURis): outcome and complication rates after the first 1000 cases. J Endourol 2009; 23: 1145-1149.

5. Michielsen DP, Coomans D, Braeckman JG, Umbrain V. Bipolar transurethral resection in saline: the solution to avoid hyponatraemia and transurethral resection syndrome. Scand J Urol Nephrol 2010; 44: 228-235.

6. Zhao C, Tang K, Yang H, Xia D, Chen Z. Bipolar Versus Monopolar Transurethral Resection of Nonmuscle-Invasive Bladder Cancer: A Meta-Analysis. J Endourol 2016; 30: 5-12.

7. Del Rosso A, Pace G, Masciovecchio S, Saldutto P, Galatioto GP, Vicentini $C$. Plasmakinetic bipolar versus monopolar transurethral resection of non-muscle invasive bladder cancer: a single center randomized controlled trial. Int J Urol 2013; 20: 399-403.

8. Venkatramani V, Panda A, Manojkumar R, Kekre NS. Monopolar versus bipolar transurethral resection of bladder tumors: a single center, parallel arm, randomized, controlled trial. J Urol 2014; 191 : 1703-1707.

9. Teoh JY, Chan ES, Yip SY, et al. Comparison of Detrusor Muscle Sampling Rate in Monopolar and Bipolar Transurethral Resection of Bladder Tumor: A Randomized Trial. Ann Surg Oncol 2017; 24: 1428-1434

10. Bolat D, Gunlusoy B, Degirmenci T, Ceylan Y, Polat S, Aydin E, Aydogdu O, Kozacioglu Z. Comparing the short-term outcomes and complications of monopolar and bipolar transurethral resection of non-muscle invasive bladder cancers: a prospective, randomized, controlled study. Arch Esp Urol 2016; 69: 225-233.

11. Sugihara T, Yasunaga $\mathrm{H}$, Horiguchi $\mathrm{H}$, et al. Comparison of perioperative outcomes including severe bladder injury between monopolar and bipolar transurethral resection of bladder tumors: a population based comparison. J Urol 2014; 192: 1355-1359.

12. Liem E, McCormack M, Chan ESY, et al. Monopolar vs. bipolar transurethral resection for non-muscle invasive bladder carcinoma: A post-hoc analysis from a randomized controlled trial. Urol Oncol 2018; 36: 338.e1-338.e11.

13. Cui Y, Chen H, Liu L, Chen J, Qi L, Zu X. Comparing the Efficiency and Safety of Bipolar and Monopolar Transurethral Resection for Non-Muscle Invasive Bladder Tumors: A Systematic Review and Meta-Analysis. J Laparoendosc Adv Surg Tech A 2016; 26: 196-202.

14. Zhou Z, Zhao S, Lu Y, Wu J, Li Y, Gao Z, Yang D, Cui Y. Meta-analysis of efficacy and safety of continuous saline bladder irrigation compared with intravesical chemotherapy after transurethral resection of bladder tumors. World J Urol 2019; 37: 1075-1084.

15. Sylvester RJ, van der Meijden AP, Oosterlinck W, Witjes JA, Bouffioux $C$, Denis L, Newling DW, Kurth K. Predicting recurrence and progression in individual patients with stage Ta T1 bladder cancer using EORTC risk tables: a combined analysis of 2596 patients from seven EORTC trials. Eur Urol 2006; 49: 466-465; discussion 475-467.

16. Naselli A, Puppo P. En Bloc Transurethral Resection of Bladder Tumors: A New Standard? J Endourol 2017; 31: S20-S24.

17. Gontero P, Sylvester R, Pisano F, et al. Prognostic factors and risk groups in T1G3 non-muscle-invasive bladder cancer patients initially treated with Bacillus Calmette-Guerin: results of a retrospective multicenter study of 2451 patients. Eur Urol 2015; 67: 74-82.

18. Kramer MW, Altieri V, Hurle R, Lusuardi L, Merseburger AS, Rassweiler J, Struck JP, Herrmann TRW. Current Evidence of Transurethral En-bloc Resection of Nonmuscle Invasive Bladder Cancer. Eur Urol Focus 2017; 3: 567-576.

19. Kramer MW, Rassweiler JJ, Klein J, et al. En bloc resection of urothelium carcinoma of the bladder (EBRUC): a European multicenter study to compare safety, efficacy, and outcome of laser and electrical en bloc transurethral resection of bladder tumor. World J Urol 2015; 33: 1937-1943. 
20. Bach T, Muschter R, Herrmann TR, et al. Technical solutions to improve the management of non-muscle-invasive transitional cell carcinoma: summary of a European Association of Urology Section for Uro-Technology (ESUT) and Section for Uro-Oncology (ESOU) expert meeting and current and future perspectives. BJU Int 2015; 115: 14-23.

21. Oh KJ, Choi YD, Chung HS, Hwang EC, Jung SI, Kwon DD, Park K, Kang TW. A novel transurethral resection technique for superficial flat bladder tumor: grasp and bite technique. Korean J Urol 2015; 56: 227-232.

22. Zare R, Grabe M, Hermann GG, Malmstrom PU. Can routine outpatient follow-up of patients with bladder cancer be improved? A multicenter prospective observational assessment of blue light flexible cystoscopy and fulguration. Res Rep Urol 2018; 10: 151-157.

23. Herrmann TR, Wolters M, Kramer MW. Transurethral en bloc resection of nonmuscle invasive bladder cancer: trend or hype. Curr Opin Urol 2017; 27: 182-190.

24. Naselli A, Introini C, Germinale F, Spina B, Puppo P. En bloc transurethral resection of bladder lesions: a trick to retrieve specimens up to $4.5 \mathrm{~cm}$. BJU Int 2012; 109: 960-963.

25. Hurle R, Lazzeri M, Colombo P, et al. „En Bloc” Resection of Nonmuscle Invasive Bladder Cancer: A Prospective Single-center Study. Urology 2016; 90: 126-130.

26. Maurice MJ, Vricella GJ, MacLennan G, Buehner P, Ponsky LE. Endoscopic snare resection of bladder tumors: evaluation of an alternative technique for bladder tumor resection. J Endourol 2012; 26 : 614-617.

27. Fritsche HM, Otto W, Eder F, et al. Water-jet-aided transurethral dissection of urothelial carcinoma: a prospective clinical study. J Endourol 2011; 25: 1599-1603.

28. Kawada T, Ebihara K, Suzuki T, Imai K, Yamanaka H. A new technique for transurethral resection of bladder tumors: rotational tumor resection using a new arched electrode. J Urol 1997; 157: 2225-2226.

29. Ukai R, Kawashita E, Ikeda H. A new technique for transurethral resection of superficial bladder tumor in 1 piece. J Urol 2000; 163: 878-879.

30. Saito S. Transurethral en bloc resection of bladder tumors. J Urol 2001; 166: 2148-2150

31. Lodde M, Lusuardi L, Palermo S, Signorello D, Maier K, Hohenfellner R, Pycha A. En bloc transurethral resection of bladder tumors: use and limits. Urology 2003; 62: 1089-1091.

32. Zhang J, Wang L, Mao S, et al. Transurethral en bloc resection with bipolar button electrode for non-muscle invasive bladder cancer. Int Urol Nephrol 2018; 50: 619-623.

33. Balan GX, Geavlete PA, Georgescu DA, et al. Bipolar en bloc tumor resection versus standard monopolar TURBT - which is the best way to go in non-invasive bladder cancer? Rom J Morphol Embryol 2018; 59: 773-780.

34. Sureka SK, Agarwal V, Agnihotri S, Kapoor R, Srivastava A, Mand hani A. Is en-bloc transurethral resection of bladder tumor for non-muscle invasive bladder carcinoma better than conventional technique in terms of recurrence and progression?: A prospective study. Indian J Urol 2014; 30: 144-149.

35. Zhang KY, Xing JC, Li W, Wu Z, Chen B, Bai DY. A novel transurethra resection technique for superficial bladder tumor: retrograde en bloc resection. World J Surg Oncol 2017; 15: 125.

36. Wu YP, Lin TT, Chen SH, et al. Comparison of the efficacy and feasibility of en bloc transurethral resection of bladder tumor versus conventional transurethral resection of bladder tumor: A meta-analysis. Medicine (Baltimore) 2016; 95: e5372.

37. Nagele U, Kugler M, Nicklas A, Merseburger AS, Walcher U, Mikuz G Herrmann TR. Waterjet hydrodissection: first experiences and short-term outcomes of a novel approach to bladder tumor resec tion. World J Urol 2011; 29: 423-427.

38. Islas-Garcia JJ, Campos-Salcedo JG, Lopez-Benjume BI, Torres Gómez JJ, Aguilar-Colmenero J, Martínez-Alonso IA, Gil-Villa SA Surgical technique for en bloc transurethral resection of bladder tumour with a Hybrid Knife((R)). Actas Urol Esp 2016; 40: 263-267.

39. Hu J. SIU 2017: En BIOC Transurethral Resection with Hybrid Knife for Treatment of Primary Non-Muscle-Invasive Bladder Cancer: A Single-Center, Randomized, Controlled Trial. UroToday 2017.
40. Gakis G, Karl A, Bertz S, et al. Transurethral en-bloc hydrodissec tion for non-muscle invasive bladder cancer: Results of a randomized controlled trial. Eur Urol Supp 2017; 16: e1143-e1144.

41. Kramer MW, Bach T, Wolters M, Imkamp F, Gross AJ, Kuczyk MA, Merseburger AS, Herrmann TR. Current evidence for transurethral laser therapy of non-muscle invasive bladder cancer. World J Urol 2011; 29: 433-442.

42. Soler-Martínez J, Vozmediano-Chicharro R, Morales-Jiménez Hernández-Alcaraz D, Vivas-Vargas E, García-Vaquero IS, Baena-González V. Holmium laser treatment for low grade, low stage, noninvasive bladder cancer with local anesthesia and early instillation of mitomycin C. J Urol 2007; 178: 2337-2339.

43. Zhu Y, Jiang X, Zhang J, Chen W, Shi B, Xu Z. Safety and efficacy of holmium laser resection for primary nonmuscle-invasive bladder cancer versus transurethral electroresection: single-center experience. Urology 2008; 72: 608-612.

44. Xishuang S, Deyong Y, Xiangyu C, et al. Comparing the safety and efficiency of conventional monopolar, plasmakinetic, and holmium laser transurethral resection of primary non-muscle invasive bladder cancer. J Endourol 2010; 24: 69-73.

45. D'Souza N, Verma A. Holmium laser transurethral resection of bladder tumor: Our experience. Urol Ann 2016; 8: 439-443.

46. Xia SJ, Zhang YN, Lu J, Sun XW, Zhang J, Zhu YY, Li WG. [Thulium laser resection of prostate-tangerine technique in treatment of benign prostate hyperplasia]. Zhonghua Yi Xue Za Zhi 2005; 85: 3225-3228.

47. Wang W, Liu H, Xia S. Thulium laser treatment for bladder cancer. Asian J Urol 2016; 3: 130-133.

48. Gao X, Ren S, Xu C, Sun Y. Thulium laser resection via a flexible cystoscope for recurrent non-muscle-invasive bladder cancer: initial clinical experience. BJU Int 2008; 102: 1115-1118.

49. Guo G, Yang Y, Hong BF, Zhang X, Zhou ZH. [Histopathologic analysis of 2 micron continuous wave laser for the partial cystectomy of bladder urothelial carcinoma]. Zhonghua Wai Ke Za Zhi 2009; 47: 1566-1568

50. Wei ZT, Xu Y, Xu F, Yang Y, Guo G, Hong BF, Zhang X. [Transurethral partial cystectomy using a 2 microm continuous wave laser in treatment of bladder carcinoma: 1-year follow-up]. Zhonghua Wai Ke Za Zhi 2010; 48: 221-223.

51. Wolters M, Kramer MW, Becker JU, et al. Tm:YAG laser en bloc mu cosectomy for accurate staging of primary bladder cancer: early experience. World J Urol 2011; 29: 429-432.

52. Yang Y, Wei ZT, Zhang X, Hong BF, Guo G. [Initial experience of partial cystectomy in treatment of bladder tumor by 2 microm continuous wave laser]. Zhonghua Wai Ke Za Zhi 2009; 47: 143-145.

53. Yang Y, Wei ZT, Zhang X, Hong BF, Guo G. Transurethral partial cystectomy with continuous wave laser for bladder carcinoma. J Urol 2009; 182: 66-69.

54. Muto G, Collura D, Giacobbe A, D'Urso L, Muto GL, Demarchi A Coverlizza S, Castelli E. Thulium:yttrium-aluminum-garnet laser for en bloc resection of bladder cancer: clinical and histopathologic advantages. Urology 2014; 83: 851-855.

55. Zhong C, Guo S, Tang Y, Xia S. Clinical observation on 2 micron laser for non-muscle-invasive bladder tumor treatment: single-center experience. World J Urol 2010; 28: 157-161.

56. Liu H, Wu J, Xue S, Zhang O, Ruan Y, Sun X, Xia S. Comparison of the safety and efficacy of conventional monopolar and 2-micron laser transurethral resection in the management of multiple nonmus cle-invasive bladder cancer. J Int Med Res 2013; 41: 984-992.

57. Chen X, Liao J, Chen L, et al. En bloc transurethral resection with 2-micron continuous-wave laser for primary non-muscle-invasive bladder cancer: a randomized controlled trial. World J Urol 2015; 33: 989-995.

58. Zhang XR, Feng C, Zhu WD, Si JM, Gu BJ, Guo H, Song LJ, Li C. Two Micrometer Continuous-Wave Thulium Laser Treating Primary Non-Muscle-Invasive Bladder Cancer: Is It Feasible? A Randomized Prospective Study. Photomed Laser Surg 2015; 33: 517-523.

59. Migliari R, Buffardi A, Ghabin H. Thulium Laser Endoscopic En Bloc Enucleation of Nonmuscle-Invasive Bladder Cancer. J Endourol 2015; 29: 1258-1262.

60. Li K, Xu Y, Tan M, Xia S, Xu Z, Xu D. A retrospective comparison of thulium laser en bloc resection of bladder tumor and plasmakinetic transurethral resection of bladder tumor in primary non-muscle invasive bladder cancer. Lasers Med Sci 2019; 34: 85-92. 
61. Zhang Z, Zeng S, Zhao J, et al. A Pilot Study of Vela Laser for En Bloc Resection of Papillary Bladder Cancer. Clin Genitourin Cancer 2017; 15: e311-e314.

62. Xu H, Ma J, Chen Z, et al. Safety and Efficacy of En Bloc Transurethral Resection With 1.9 microm Vela Laser for Treatment of Non-Muscle-invasive Bladder Cancer. Urology 2018; 113: 246-250.

63. Tao W, Yang D, Shan Y, Xue B, Sun C, Zang Y, Zhang Y. Safety and efficacy of 120W high performance system greenlight laser vaporization for non-muscle-invasive bladder cancer. I Xray Sci Technol 2013; 21: 309-316.

64. He D, Fan J, Wu K, et al. Novel green-light KTP laser en bloc enucleation for nonmuscle-invasive bladder cancer: technique and initial clinical experience. J Endourol 2014; 28: 975-979.

65. Chen J, Zhao Y, Wang S, Jin X, Sun P, Zhang L, Wang M. Green-light laser en bloc resection for primary non-muscle-invasive bladder tumor versus transurethral electroresection: A prospective, nonrandomized two-center trial with 36-month follow-up. Lasers Surg Med 2016; 48: 859-865.

\section{Address for correspondence}

\section{Wojciech Krajewski}

Department of Urology and Oncological Urology

Wroclaw Medical University

213 Borowska St.

50-556 Wrocław, Poland

e-mail: wk@softstar.pl

Submitted: 29.04 .2019

Accepted: 4.08 .2019 